\title{
Dynamic Crack Propagation Problems of Fibre-Reinforced Concrete
}

\author{
Y. H. Cheng, ${ }^{1}$ Lu Nian Chun, ${ }^{2}$ and Li Xin-gang ${ }^{3}$ \\ ${ }^{1}$ School of Resources \& Civil Engineering, Northeastern University, Shenyang 110004, China \\ ${ }^{2}$ School of Materials, Shenyang Ligong University, Shenyang 110168, China \\ ${ }^{3}$ School of Civil Engineering, Harbin Engineering University, Harbin 150001, China \\ Correspondence should be addressed to Y. H. Cheng, neu_cyh@163.com
}

Received 28 June 2011; Accepted 17 October 2011

Academic Editor: Muhammad Hadi

Copyright (๑) 2012 Y. H. Cheng et al. This is an open access article distributed under the Creative Commons Attribution License, which permits unrestricted use, distribution, and reproduction in any medium, provided the original work is properly cited.

Applying the built dynamic crack model of fibre concrete, bridging fiber segment is substituted by loads. When a crack propagates its fiber continues to break. By the approaches of the theory of complex functions, the problems dealt with can be translated into Riemann-Hilbert problems. Analytical solutions of the displacements, stresses, and dynamic stress intensity factors under the action of of moving variable loads $P x^{2} / t^{2}$ and $P t^{3} / x^{2}$, respectively, are attained by the ways of self-similar measures. After those analytical solutions are utilized by superposition theorem, the solutions of arbitrary complex problems can be obtained.

\section{Introduction}

Regarding the enhancing mechanism of fiber-reinforced concrete, there exist two popular theories $[1,2]$ : the theory of composite materials and the theory of fibrous space.

According to the theory of composite materials, fiberreinforced concrete is regarded as a multiphase system, that is, the fiber and the concrete separately. The properties of the composite materials are the sum of each ones, and basic assumptions are as follows.

(1) Fibers were distributed continuously and uniformly, and the directions were consistent with the force.

(2) Fiber and matrix bond well, that is, they have the same strain, and no relative sliding occurred.

(3) Fiber and matrix are elastic deformation, and transverse deformation is the same.

In the light of disorder status of the short steel fiberreinforced concrete, theory of fibrous space is put forward. This theory derives from the theorem of linear elastic fracture mechanics, and fiber space is referred to as the enhancing mechanism of the basic parameters. When fibers are mixed in concrete, they can restrict the crack propagation effectively. The smaller the fiber space is, the greater the stress concentration abates; therefore the strength and toughness of concrete increase easily.

Because fiber mixed into concrete is capable of resisting crack formation and expansion, there is obvious difference in fracture behavior and crack resistance between fiberreinforced concrete and general concrete. Therefore, fracture characteristic of fiber-reinforced concrete was studied and its fracture model was built, which processes the great significance not only for understanding fiber-reinforced concrete material itself, but also for analyzing structural performance [3].

\section{Fracture of Fiber-Reinforced Concrete}

Fiber reinforced concrete with fibrous disorder distribution can be regarded as isotropic materials [4]. According to engineering practice, tension and compression are very common, so the assumption of fiber reinforced concrete crack is mode I crack.

When fiber-reinforced concrete was subjected to external loads, a fracture process zone appeared in the front of the main crack tips, and properties in fracture zone have a significant impact on fracture and toughness of fiberreinforced concrete. After fiber-reinforced concrete starts to crack, crack tip opening was prevented by bridging fibers [3]. 


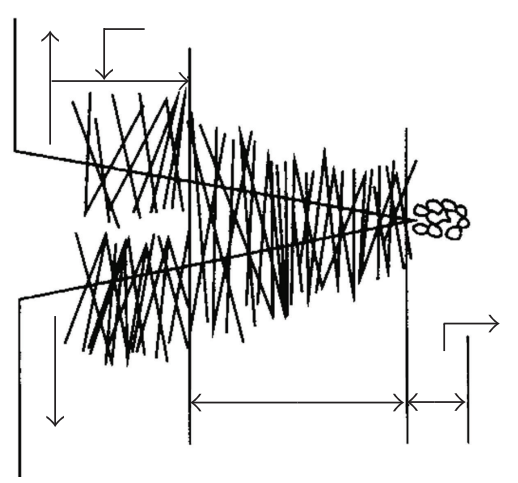

FIGURE 1: Sketch of fracture of fiber reinforced concrete.

In order to analyze fracture process of fiber-reinforced concrete, a crack model of bridging fibers in concrete was put forward [5-8]. In order to analyze fracture process of fiber-reinforced concrete, a crack model of bridging fibers in concrete was put forward [5-8], as depicted in Figure 1.

This crack model will be divided into three regions. Region A is the fracture section without stress transfer, and the fibers present pullout or break. Region B is regarded as bridging fiber zone which is also known as pseudoplastic zone and begins to fracture, while the fibers can arrest crack propagation; the length of the crack increases slowly and reaches critical state finally. Region $\mathrm{C}$ is called the microcrack zone or transition zone, and when the loads aggrandize gradually, region $\mathrm{C}$ will transform into region $\mathrm{B}$.

At bridging fiber segment, some fibers across the crack were regarded as closed impact forces which act at the crack surfaces. Bridging fibers reduced the stress intensity factors of the crack tips, and under the conditions of the same fracture toughness of the matrix material, the greater loads needed can induce crack propagation and cause material damage [9].

Bridging fibers play a vital role in the course of crack arrest, enhancement, and increasing toughness of concrete, especially when the concrete occurs crack, the role of bridging fiber cannot be ignored. Therefore, bridging fiber problems of fiber reinforced concrete are not only an important research task but also a frontline in the field.

\section{Dynamic Fracture Model of Fiber Reinforced Concrete}

During the form and propagation of the crack, bridging fibers appears. That is to say, when the crack propagates, the case of bridge still exists. Accordingly, it is an important meaning to establish an appropriate dynamic model of bridging fibers in studies on fracture problem of fiber reinforced concrete.

If the fiber failure is governed by maximum tensile stress, which occurs at the crack plane, the fiber breaks and hence the crack extension should appear in a self-similar fashion. The fiber breaks lie along a transverse line and therefore present a " $V$ " notch. The crack is supposed to nucleate an infinitesimally small microcrack situated along the $x$-axis in the format of self-similarity with the high-speed

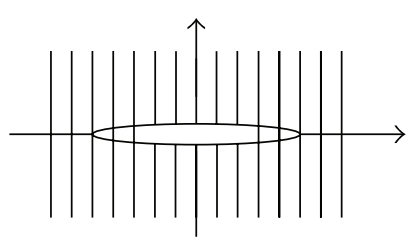

FIGURE 2: Sketch of crack extension of bridging fibers in fiber reinforced concrete.

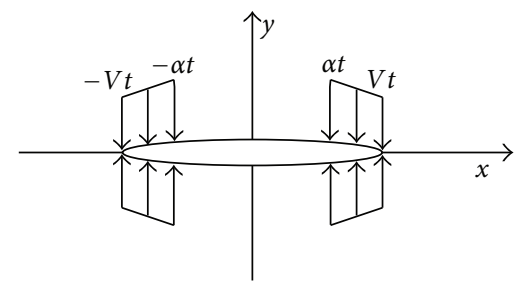

Figure 3: The dynamic model of the crack of bridging fibers in fiber reinforced concrete.

propagation, and to move symmetrically in the positive and negative $x$ directions with the constant crack tip velocity $V$ in the matrix. That is to say, the crack begins to run symmetrically along the positive and negative directions of $x$-axes from the length of zero. The crack moves at the constant velocity $V$ in the matrix of reinforced concrete. The fibers do not fracture in the vicinity of the crack tip, but the others break at the central region of crack. When crack propagates, fibers continuously break with the constant velocity $\alpha$ according to one of the authors presumption as displayed in Figure 2. Since the configuration shown in Figure 2 is symmetry both in geometry and loading about $y$-axis, only the right half-plane of the region needs to be considered for analysis. The fibers and the matrix are taken to be linearly elastic. It is further postulated that the fibers have a much higher elastic modulus in the axial direction than the matrix and therefore the fibers are taken as supporting all of the axial loads in fiber rein-forced concrete. Load is transferred between adjacent fibers through the matrix of concrete by a straightforward shear mechanism. In order to analyze conveniently, the fiber fracture lie is assumed to appear along a single plane. That is, the fiber fracture can be self-similar fiber fracture and therefore present a "V" notch. The zone of crack or notch is $y=0,|x|<V t$, and the interval of bridging fibers is $y=0, \alpha t<|x|<V t$, while the segment of fracture is $|x|<\alpha t$.

Obviously, the dynamic model of crack propagation problem of concrete in Figure 2 is illustrated by that, in Figure 3, this is a model of symmetrical crack extension, running with constant velocity $V$ in both the positive and negative directions of $x$-axis, so are the locations of bridging fibers fracturing with constant velocity $\alpha$. The intervals of bridging fibers have the symmetrical state with respect to $x$-axis. Each bridging fiber is replaced by a pair of vertical traction forces which act at the points with the same $x$ coordinate on the upper and lower crack surfaces, but in an opposite direction. Each traction force is postulated to 
be balanced with the load of fracture of a fiber from the matrix of concrete. The present model has the symmetries of geometrical and mechanical conditions with respect to the $x$ - and $y$-axes. At $y=0$, traction forces act at the section of $\alpha t<|x|<V t$, which represent fibrous compressive stress, whereas they do not act on the rest. Fibers in rein-forced concrete are usually arranged tightly; therefore compressive stress produced by bridging fiber traction forces distribute sequentially. For example, at $y=0$, in the zone of $\alpha t<x<$ $V t$, the vertical displacement of the crack edges is not the same, hence the bridging fiber traction forces are not equal. It is obvious that traction forces are larger near the points of $\alpha t$ (where the fibers have broken) and they are smaller close to the points of $V t$. Accordingly bridging fiber traction forces postulated have relation to the dimension of displacement $x$. On the other hand, when a crack propagates at high speed, its length will increase with time $t$. The longer a crack moves, the more fibers fracture. That is to say, the number of broken fibers relates to time $t$. Bridging fiber traction forces are thought to be unlike at the interval of $y=0, \alpha t<|x|<V t$. In the above analysis, the fibres in the matrix of concrete are supposed to be distributed homogenously. Each fibre has the same strength. When the fracture occurs the fibre and the matrix are in the same plane of crack extension. Certainly, this is a postulated mechanical model which may not accord with real situations, and it waits for more improvements aftertime.

\section{The Correlative Formulas of Self-Similar Functions}

In order to solve efficiently fracture dynamics problems of composite materials, solutions will be obtained under the action of variable loads for mode I moving crack. According to the theorem of generalized functions, the different boundary condition problems considered will be translated into Keldysh-Sedov mixed boundary value problem by means of self-similar functions, and the corresponding solutions will be attained.

Suppose at $y=0$ there are any number of loaded sections and displacement sections along the $x$-axis, and the ends of these sections are moving with different constant velocity. At the initial moment $t=0$ the half-plane is at rest. In these sections the loads and displacements are arbitrary linear combination of the following functions [10-13]:

$$
\frac{d^{k} f_{k_{1}}(x)}{d x^{k}} \cdot \frac{d^{s} f_{s_{1}}(t)}{d t^{s}}
$$

where

$$
f_{i}(\xi)= \begin{cases}0 & \xi<0 \\ \xi^{i} & \xi>0\end{cases}
$$

Here $k, k_{1}$, and $s, s_{1}$ are arbitrary integer positive numbers.

An arbitrary continuous function of two variables $x$ and $t$ may be expressed as a linear superposition of (1); thus it has a significance in principle to seek the loads or the displacements satisfying the type of (2). Let us introduce the following linear differential operator as well as integral operator:

$$
L=\frac{\partial^{m+n}}{\partial x^{m} \partial t^{n}}, \quad \text { inverse }: L^{-}=\frac{\partial^{-m-n}}{\partial x^{-m} \partial t^{-n}} .
$$

Here $+m+n,-m-n$, and 0 represent the $(m+n)$ th order derivative, the $(m+n)$ th order integral, and function's self. It is easy to testify that there exist constants $m$ and $n$. When substituting (3) into (2), (1), we will obtain functions that are homogeneous functions of $x$ and $t$ of zeroth dimension (homogeneous), and the couple $m, n$ will be called an index of self-similarity [11-13].

Utilizing correlative representations of elastodynamics equations of motion for an orthotropic anisotropic body [10-13].

For the case when functions $L u$ and $L v$ are homogeneous

$$
u^{0}=L u, \quad v^{0}=L v, \quad \sigma_{y}^{0}=L \sigma_{y},
$$

for the case when functions $L \sigma_{y}$ and $L \tau_{x y}$ are homogeneous,

$$
u^{0}=\frac{\partial}{\partial t} L u, \quad v^{0}=\frac{\partial}{\partial t} L v, \quad \sigma_{y}^{0}=\frac{\partial}{\partial t} L \sigma_{y} .
$$

The relative self-similar functions are as follows [10-13]:

$$
\begin{gathered}
\sigma_{y}^{0}=\left(\frac{1}{t}\right) \operatorname{Re} F(\tau), \quad v^{0}=\operatorname{Re} W(\tau), \\
W^{\prime}(\tau)=\left[\frac{D_{1}(\tau)}{D(\tau)}\right] F(\tau),
\end{gathered}
$$

where $F(\tau), \mathrm{W}(\tau)$ are self-similar functions, and the relation is the same as (7). The values of $D_{1}(\tau) / D(\tau)$ can be determined from Appendix of literature $[11,13]$ :

$$
\begin{aligned}
& \frac{D(\tau)}{D_{1}(\tau)} \\
& \quad=\frac{i \rho C_{1}\left[\left(2 C_{2}^{2}-\tau^{2}\right)^{2}-4 C_{2}^{3} C_{1}^{-1} \sqrt{\left(C_{1}^{2}-\tau^{2}\right)\left(C_{2}^{2}-\tau^{2}\right)}\right]}{\tau^{2} \sqrt{C_{1}^{2}-\tau^{2}}}
\end{aligned}
$$

here indicated only: $D_{1}(\tau) / D(\tau)$ in the range of the subsonic speeds is purely imaginary for the values which we are considering. Thus, elastodynamics problems for an orthotropic anisotropic body investigated can be transformed into seeking the single unknown function problems on $F(\tau)$ and $W(\tau)$ meeting the boundary value conditions. In the general case this is Riemann-Hilbert problem in the theory of complex functions (in the simplest cases we have KeldyshSedov or Dirichlet problem), and this kind of problem is easily settled by the usual methods, such as Muskhelishvili $[14,15]$.

Fracture dynamics problems will be studied for an infinite orthotropic anisotropic body. Assume at the initial moment $t=0$ a crack appears at the origin of coordinates 
and begins spreading symmetrically at constant velocity $V$ (for the subsonic speeds) in both the positive and negative directions of $x$-axis, respectively, and at $t<0$, the half-plane was at rest. The crack surfaces are subjected to the different types of loads under the plane strain states.

\section{The Solutions of Idiographic Problems}

In order to resolve efficaciously symmetrical dynamics problems with bridging fibers of fiber reinforced concrete, solutions will be found under the conditions of unlike loads for mode I running crack. In the light of the theorem of generalized functions, the different boundary condition problems investigated will be changed into Keldysh-Sedov mixed boundary value problem by the methods of selfsimilar functions, and the corresponding solutions will be acquired. The problems studied are under the plane strain states.

(1) Postulate, at the initial moment $t=0$, a microcrack abruptly appears at the coordinate origin and begins expanding symmetrically with constant velocity in the positive and negative directions of $x$-axis, respectively. The surfaces of the crack are subjected to normal point force $P x^{2} / t^{2}$, moving at a constant velocity $\beta$ along the positive direction of $x$-axis, where $\beta<V$; at $t<0$ the half-plane was at rest. The boundary conditions of the problem will be written as

$$
\begin{gathered}
\sigma_{y}(x, 0, t)=-\frac{P x^{2}}{t^{2}} \cdot \delta(x-\beta t), \quad|x|<V t \\
v(x, 0, t)=0, \quad|x|>V t .
\end{gathered}
$$

In this case the displacement will evidently be homogeneous functions, in which, $L=1$. Utilizing $\tau=x / t$, the theory of generalized functions [16-18] as well as (4) and (6), the first representation of (9) can be written:

$$
\operatorname{Re} F(\tau)=-\frac{P x^{2}}{t^{2}} \cdot t \delta(x-\beta t)=-P \tau^{2} \delta(\tau-\beta), \quad|\tau|<V
$$

In terms of (4), (6), (7), boundary conditions (10) will be further rewritten:

$$
\begin{gathered}
\operatorname{Re}\left[\frac{W^{\prime}(\tau) D(\tau)}{D_{1}(\tau)}\right]=-P \tau^{2} \delta(\tau-\beta), \quad|\tau|<V, \\
\operatorname{Re}\left[W^{\prime}(\tau)\right]=0, \quad|\tau|>V .
\end{gathered}
$$

Deducting from the above formulas, the solution of $W^{\prime}(\tau)$ must have the format:

$$
W^{\prime}(\tau)=\xi(\tau) \cdot \frac{\tau^{2}}{(\tau-\beta)} .
$$

In the formula $\xi(\tau)$ has no singularity in the zone of $|\tau|<$ $V$, while $D(\tau) / D_{1}(\tau)$ is purely imaginary for the subsonic speeds; therefore $\xi(\tau)$ must be purely real in the section of $|\tau|<V$. Thus, question (11) becomes

$$
\begin{array}{ll}
\operatorname{Re} \xi(\tau)=0, & |\tau|>V, \\
\operatorname{Im} \xi(\tau)=0, & |\tau|<V .
\end{array}
$$

According to symmetry and the conditions of the infinite point of the plane corresponding to the origin of coordinates of the physical plane as well as singularities of the crack tip $[19,20]$, the unique solution of the Keldysh-Sedov problem (13) can be gained:

$$
\xi(\tau)=A\left(V^{2}-\tau^{2}\right)^{-1 / 2}
$$

where $A$ is an unknown constant.

Substituting (14) into (12) and (7), we can obtain

$$
\begin{gathered}
W^{\prime}(\tau)=\frac{A \tau^{2}}{(\tau-\beta)\left(V^{2}-\tau^{2}\right)^{1 / 2}}, \\
F(\tau)=\frac{A \tau^{2}}{(\tau-\beta)\left(V^{2}-\tau^{2}\right)^{1 / 2}} \cdot \frac{D(\tau)}{D_{1}(\tau)} .
\end{gathered}
$$

Then substituting (15) into (11), at $\tau \rightarrow \beta$, constant $A$ can be confirmed:

$$
A=\frac{-P \sqrt{V^{2}-\beta^{2}}}{\pi \operatorname{Im}\left[D(\beta) / D_{1}(\beta)\right]} .
$$

Then putting (15) into (6) and (4), at the surface $y=$ 0 , we will attain the stress and the stress intensity factor, respectively,

$$
\sigma_{y}=\frac{A \tau^{2}}{(\tau-\beta) \sqrt{x^{2}-V^{2} t^{2}}} \cdot \operatorname{Im}\left[\frac{D(\tau)}{D_{1}(\tau)}\right]
$$$$
|x|>V t
$$

$$
K_{\mathrm{I}}(t)=-\frac{A V}{V-\beta} \sqrt{\frac{\pi V}{t}} \cdot \operatorname{Im}\left[\frac{D(V)}{D_{1}(V)}\right] .
$$

The first of (15) can be rewritten as

$$
\begin{aligned}
W^{\prime}(\tau) & =\frac{A \tau^{2}}{(\tau-\beta)\left(V^{2}-\tau^{2}\right)^{1 / 2}} \\
& =\left(\tau+\beta+\frac{\beta^{2}}{\tau-\beta}\right) \frac{A}{\left(V^{2}-\tau^{2}\right)^{1 / 2}}
\end{aligned}
$$

Integrating (18), one will gain $W(\tau)$. But it has three terms, separate denotation is more expedient, then putting them into (4), (6), (7), and integral formulae can be utilized 
in literature [21], then the divisional displacements $v_{1}, v_{2}, v_{3}$ can be gained, respectively,

$$
\begin{aligned}
& v_{1}=v_{1}^{0}=\operatorname{Re} W_{1}(\tau)=\operatorname{Re} \int_{\infty}^{x / t} \frac{A \tau}{\sqrt{V^{2}-\tau^{2}}} d \tau \\
& =-\left.A \operatorname{Re} \sqrt{V^{2}-\tau^{2}}\right|_{\infty} ^{x / t}=-\frac{A}{t} \sqrt{V^{2} t^{2}-x^{2}}, \quad|x|<V t, \\
& v_{2}=v_{2}{ }^{0}=\operatorname{Re} W_{2}(\tau)=\operatorname{Re} \int_{\infty}^{x / t} \frac{A \beta}{\sqrt{V^{2}-\tau^{2}}} d \tau \\
& =\left.A \beta \cdot \operatorname{Re} \arcsin \frac{\tau}{V}\right|_{\infty} ^{x / t}=A \beta \arcsin \frac{x}{V t} \quad|x|<V t, \\
& v_{3}=v_{3}{ }^{0}=\operatorname{Re} W_{3}(\tau)=\operatorname{Re} \int_{\infty}^{x / t} \frac{A \beta^{2}}{(\tau-\beta) \sqrt{V^{2}-\tau^{2}}} d \tau \\
& =\frac{A \beta^{2}}{\sqrt{V^{2}-\beta^{2}}} \\
& \times\left.\operatorname{Re} \ln \left|\frac{V^{2}-\beta \tau-\sqrt{\left(V^{2}-\beta^{2}\right)\left(V^{2}-\tau^{2}\right)}}{V(\beta-\tau)}\right|\right|_{\infty} ^{x / t} \\
& =\frac{A \beta^{2}}{\sqrt{V^{2}-\beta^{2}}} \\
& \times \ln \left|\frac{V^{2} t-\beta x-\sqrt{\left(V^{2}-\beta^{2}\right)\left(V^{2} t^{2}-x^{2}\right)}}{V(\beta t-x)}\right|, \\
& |x|<V t .
\end{aligned}
$$

The displacement $v$ is the sum of subdistrict displacement: $v=v_{1}+v_{2}+v_{3}$. After the addition of (19), the displacement $v$ is gained:

$$
\begin{aligned}
& v=A[-\frac{\sqrt{V^{2} t^{2}-x^{2}}}{t}+\beta \arcsin \frac{x}{V t}+\frac{\beta^{2}}{\sqrt{V^{2}-\beta^{2}}} \\
&\left.\times \ln \left|\frac{V^{2} t-\beta x-\sqrt{\left(V^{2}-\beta^{2}\right)\left(V^{2} t^{2}-x^{2}\right)}}{V(\beta t-x)}\right|\right] \\
& \quad|x|<V t .
\end{aligned}
$$

By means of the solution of (20), the bridging fibrous fracture speeds of fiber concrete $\alpha$ can be acquired:

$$
\begin{array}{r}
\Delta=A\left[-\sqrt{V^{2}-\alpha^{2}}+\beta \arcsin \frac{\alpha}{V}+\frac{\beta^{2}}{\sqrt{V^{2}-\beta^{2}}}\right. \\
\left.\quad \times \ln \left|\frac{V^{2}-\beta \alpha-\sqrt{\left(V^{2}-\beta^{2}\right)\left(V^{2}-\alpha^{2}\right)}}{V(\beta-\alpha)}\right|\right] \\
|x|=\alpha t,
\end{array}
$$

where $\Delta$ is ascertained by single axis tensile test of bridging fibers of fiber reinforced concrete, while $V, \beta, t$ are regarded as known constants, respectively, then fibrous fracture velocity $\alpha$ obtained is numerical solution according to (21); therefore the problem on the dynamic crack model of bridging fibers in fiber concrete is solved.

(2) Presume that the rest conditions are the same as those in the above ensample except that the applied loads become an increasing load $P t^{3} / x^{2}$. The boundary conditions will be as

$$
\begin{gathered}
\sigma_{y}(x, 0, t)=-\frac{P t^{3}}{x^{2}} \cdot \delta(x-\beta t), \quad|x|<V t \\
v(x, 0, t)=0, \quad|x|>V t .
\end{gathered}
$$

In this case the stress will apparently be homogeneous functions, in which $L=1$. According to (5), (6), and the theory of generalized functions [16-18], the first expression of the boundary condition (22) can be written as follows:

$$
\begin{aligned}
\operatorname{Re}[F(\tau)] & =-\left(\frac{3 P t^{2}}{x^{2}}\right) t \delta(x-\beta t) \\
& =-3 P \tau^{-2} \delta(\tau-\beta), \quad|\tau|<V .
\end{aligned}
$$

At $x \neq \beta t$, the derivative of $\delta(x-\beta t)$ is zero, then the result will be gained.

In the light of (5), (6), (7), boundary conditions (23) will be further rewritten:

$$
\begin{aligned}
\operatorname{Re}\left[\frac{W^{\prime}(\tau) D(\tau)}{D_{1}(\tau)}\right] & =-3 P \tau^{-2} \delta(\tau-\beta), \quad|\tau|<V, \\
\operatorname{Re}\left[W^{\prime}(\tau)\right] & =0, \quad|\tau| .>V .
\end{aligned}
$$

From the above formulas, the unique solution of $W^{\prime}(\tau)$ can be facilely deduced:

$$
W^{\prime}(\tau)=\frac{\xi(\tau)}{\left[\tau^{2}(\tau-\beta)\right]} .
$$

In the formulae, $\xi(\tau)$ has no singularity in the domain of $-V_{2}<\tau<V_{1}$, while $D(\tau) / D_{1}(\tau)$ is purely imaginary for the subsonic speeds; therefore $\xi(\tau)$ must be purely real at the interval of $-V_{2}<\tau<V_{1}$. Thus, question (24) takes

$$
\begin{aligned}
\operatorname{Re} \xi(\tau)=0, & & |\tau|>V, \\
\operatorname{Im} \xi(\tau)=0, & & |\tau|<V .
\end{aligned}
$$

In terms of symmetry and the conditions of the infinite point of the plane corresponding to the origin of coordinates of the physical plane as well as singularities of the crack tip $[19,20]$, the unique solution of the Keldysh-Sedov problem (26) can be obtained:

$$
\xi(\tau)=A\left(V^{2}-\tau^{2}\right)^{-3 / 2},
$$

where $A$ is an unbeknown constant.

Then putting (27) into (25) and (7) results in

$$
\begin{gathered}
W^{\prime}(\tau)=\frac{A}{\tau^{2}(\tau-\beta)\left(V^{2}-\tau^{2}\right)^{3 / 2}}, \\
F(\tau)=\frac{A}{\tau^{2}(\tau-\beta)\left(V^{2}-\tau^{2}\right)^{3 / 2}} \cdot \operatorname{Im}\left[\frac{D(\tau)}{D_{1}(\tau)}\right] .
\end{gathered}
$$


Substituting (28) into (24), at $\tau \rightarrow \beta$, constant $A$ can be ascertained:

$$
A=-\frac{3 P\left(V^{2}-\beta^{2}\right)^{3 / 2}}{\pi \operatorname{Im}\left[D(\beta) / D_{1}(\beta)\right]} .
$$

In an orthotropic isotropic body, the disturbance scope of elastic wave can be illuminated by the circular area of radius $c_{1} t$ and $c_{2} t$. In an orthotropic anisotropic body, the disturbance range of elastic wave is not the circular area and can not exceed threshold value $C_{d}=\sqrt{C_{11} / \rho}$ of elastic body, where $C_{11}$ is an elastic constant of materials. At $|x|>$ $C_{d} t$, with $\operatorname{Im}\left[D_{1}(\tau) / D(\tau)\right]=0$, thus the stresses and the displacements are zero which coincide with initial boundary conditions; and this shows that disturbance of elastic wave cannot overrun $C_{d} t$.

Now inserting (28) into (5), (6), and (7), at the surface $y=0$, the stresses and the dynamic stress intensity factor are gained, respectively,

$$
\begin{aligned}
\sigma_{y}= & \operatorname{Re} \int_{\infty}^{x / t} \frac{A \cdot \operatorname{Im}\left[D(\tau) / D_{1}(\tau)\right]}{\tau^{3}(\tau-\beta)\left(V^{2}-\tau^{2}\right)^{3 / 2}} d \tau, \quad|x|>V t \\
K_{I}^{(1)}(t)= & \lim _{x \rightarrow V t} \sqrt{2 \pi(x-V t)} \\
& \cdot \operatorname{Re} \int_{\infty}^{x / t} \frac{A \cdot \operatorname{Im}\left[D(\tau) / D_{1}(\tau)\right]}{\tau^{3}(\tau-\beta)\left(V^{2}-\tau^{2}\right)^{3 / 2}} d \tau . \\
= & -\frac{A \sqrt{\pi t} \operatorname{Im}\left[D(V) / D_{1}(V)\right]}{V^{9 / 2}(V-\beta)} .
\end{aligned}
$$

The limit of the above belongs to the modality $0 \cdot \infty$, which should be translated into the type of $\infty / \infty$, then the aftermath of the above formula can be worked out by the approaches of L'Hospital theorem [22].

The first of (28) can be rewritten as follows:

$$
\begin{aligned}
W^{\prime}(\tau) & =\frac{A}{\tau^{2}(\tau-\beta)\left(V^{2}-\tau^{2}\right)^{1 / 2}} \\
& =\frac{1}{\beta}\left[-\frac{1}{\tau \beta}+\frac{1}{(\tau-\beta) \beta}-\frac{1}{\tau^{2}}\right] \frac{A}{\left(V^{2}-\tau^{2}\right)^{3 / 2}} .
\end{aligned}
$$

After integrating the first term of (31) with respect to variable $\tau, W(\tau)$ can be attained according to literature [23] as follows:

$$
\begin{aligned}
W_{1}(\tau) & =\int W_{1}^{\prime}(\tau) d \tau=\frac{1}{\beta} \int-\frac{1}{\tau \beta} \frac{A}{\left(V^{2}-\tau^{2}\right)^{3 / 2}} d \tau \\
& =-\frac{A}{\beta^{2}} \int \frac{A}{\tau\left(V^{2}-\tau^{2}\right)^{3 / 2}} d \tau \\
& =-\frac{A}{V^{2} \beta^{2}}\left[\frac{1}{\sqrt{V^{2}-\tau^{2}}}-\frac{1}{V} \ln \frac{V+\sqrt{V^{2}-\tau^{2}}}{\tau}\right] .
\end{aligned}
$$

The crack propagates along the $x$-axis, so $W(\tau)$ can be performed in the definite integral, and we take constant $C=0$. Then putting (32) into (6), (5), the subdistrict displacement $v_{1}$ is attained as

$$
\begin{aligned}
v_{1}= & \operatorname{Re} \int_{0}^{t} W_{1}(\tau) d \tau \\
= & \operatorname{Re} \int_{\infty}^{x / t}-\frac{A}{V^{2} \beta^{2}} \\
& \cdot\left[\frac{1}{\sqrt{V^{2}-\tau^{2}}}-\frac{1}{V} \ln \frac{V+\sqrt{V^{2}-\tau^{2}}}{\tau}\right] \cdot\left(\frac{-x}{\tau^{2}}\right) d \tau \\
= & \left.\frac{A x}{V^{3} \beta^{2}} \operatorname{Re}\left[\frac{-\sqrt{V^{2}-\tau^{2}}}{V \tau}-\left(\frac{\sqrt{V^{2}-\tau^{2}}}{V \tau}\right)\right]\right|_{\infty} \\
= & \left.\frac{A x}{V^{3} \beta^{2}} \operatorname{Re}\left[-\frac{1}{\tau} \ln \frac{V+\sqrt{V^{2}-\tau^{2}}}{\tau}\right)\right] \\
= & \left.-\frac{2 A}{V^{4} \beta^{2}} \sqrt{V^{2} t^{2}-x^{2}+\frac{\tau^{2}}{V^{3} \beta^{2}}}+\frac{1}{\tau} \ln \mid \frac{V+\sqrt{V^{2}-\tau^{2}}}{\tau}\right]\left.\right|_{\infty} ^{x / t}|x|<V t .
\end{aligned}
$$

After integrating the second nape of (31) with respect to variable $\tau, W_{2}(\tau)$ can be obtained according to literature [23] as follows:

$$
\begin{aligned}
& W_{2}(\tau)=\int W_{2}^{\prime}(\tau) d \tau=\int \frac{A}{\beta^{2}(\tau-\beta)\left(V^{2}-\tau^{2}\right)^{3 / 2}} d \tau \\
& =\frac{A}{\beta^{2}} \int \frac{(\tau+\beta)}{\left(\tau^{2}-\beta^{2}\right)\left(V^{2}-\tau^{2}\right)^{3 / 2}} d \tau \\
& =\frac{A}{\beta^{2}}\left[\frac{1}{\left(V^{2}-\beta^{2}\right) \sqrt{V^{2}-\tau^{2}}}\right. \\
& +\frac{1}{2 \sqrt{\left(V^{2}-\beta^{2}\right)^{3}}} \ln \left|\frac{\sqrt{V^{2}-\beta^{2}}-\sqrt{V^{2}-\tau^{2}}}{\sqrt{V^{2}-\beta^{2}}+\sqrt{V^{2}-\tau^{2}}}\right| \\
& +\frac{\beta \tau}{V^{2}} \frac{1}{\left(V^{2}-\beta^{2}\right) \sqrt{V^{2}-\tau^{2}}}+\frac{1}{2 \sqrt{\left(V^{2}-\beta^{2}\right)^{3}}} \\
& \left.\times \ln \left|\frac{\sqrt{V^{2}-\beta^{2}} \tau-\sqrt{V^{2}-\tau^{2}} \beta}{\sqrt{V^{2}-\beta^{2}} \tau+\sqrt{V^{2}-\tau^{2}} \beta}\right|\right] \\
& =\frac{A}{\beta^{2}\left(V^{2}-\beta^{2}\right)} \\
& \left\{\left(1+\frac{\beta \tau}{V^{2}}\right) \frac{1}{\sqrt{V^{2}-\tau^{2}}}+\frac{1}{2 \sqrt{V^{2}-\beta^{2}}}\right.
\end{aligned}
$$




$$
\begin{gathered}
\times\left[\ln \left|\frac{\sqrt{V^{2}-\beta^{2}}-\sqrt{V^{2}-\tau^{2}}}{\sqrt{V^{2}-\beta^{2}}+\sqrt{V^{2}-\tau^{2}}}\right|\right. \\
\left.\left.\quad+\ln \left|\frac{\sqrt{V^{2}-\beta^{2}} \tau-\sqrt{V^{2}-\tau^{2}} \beta}{\sqrt{V^{2}-\beta^{2}} \tau+\sqrt{V^{2}-\tau^{2}} \beta}\right|\right]\right\}+C .
\end{gathered}
$$

The crack moves along the $x$-axis, so $W_{2}(\tau)$ can be performed in the definite integral, and we take constant $C=$ 0 . Then putting (34) into (6), (5), the zonal displacement $v_{1}$ is gained as

$$
\begin{aligned}
& v_{2}=\operatorname{Re} \int_{0}^{t} W_{2}(\tau) d t=\operatorname{Re} \int_{\infty}^{x / t} W_{2}(\tau) \cdot\left(\frac{-x}{\tau^{2}}\right) d \tau \\
& =\operatorname{Re} \int_{\infty}^{x / t} \frac{A}{\beta^{2}\left(V^{2}-\beta^{2}\right)} \\
& \left\{\left(1+\frac{\beta \tau}{V^{2}}\right) \frac{1}{\sqrt{V^{2}-\tau^{2}}}+\frac{1}{2 \sqrt{V^{2}-\beta^{2}}}\right. \\
& \times\left[\ln \left|\frac{\sqrt{V^{2}-\beta^{2}}-\sqrt{V^{2}-\tau^{2}}}{\sqrt{V^{2}-\beta^{2}}+\sqrt{V^{2}-\tau^{2}}}\right|\right. \\
& \left.\left.+\ln \left|\frac{\sqrt{V^{2}-\beta^{2}} \tau-\sqrt{V^{2}-\tau^{2}} \beta}{\sqrt{V^{2}-\beta^{2}} \tau+\sqrt{V^{2}-\tau^{2}} \beta}\right|\right]\right\}\left(\frac{-x}{\tau^{2}}\right) d \tau \\
& =\frac{-A x}{\beta^{2}\left(V^{2}-\beta^{2}\right)} \\
& \times \operatorname{Re}\left\{\frac{-\sqrt{V^{2}-\tau^{2}}}{V^{2} \tau}-\frac{\beta}{V^{3}} \ln \left|\frac{V+\sqrt{V^{2}-\tau^{2}}}{\tau}\right|\right. \\
& +\frac{1}{2 V \beta} \ln \left|\frac{V+\sqrt{V^{2}-\tau^{2}}}{V-\sqrt{V^{2}-\tau^{2}}}\right| \\
& -\frac{1}{2 \sqrt{V^{2}-\beta^{2}}}\left(\frac{1}{\tau}-\frac{1}{\beta}\right) \\
& \times\left[\ln \left|\frac{\sqrt{V^{2}-\beta^{2}}-\sqrt{V^{2}-\tau^{2}}}{\sqrt{V^{2}-\beta^{2}}+\sqrt{V^{2}-\tau^{2}}}\right|\right. \\
& \left.\left.+\ln \left|\frac{\sqrt{V^{2}-\beta^{2}} \tau-\sqrt{V^{2}-\tau^{2}} \beta}{\sqrt{V^{2}-\beta^{2}} \tau+\sqrt{V^{2}-\tau^{2}} \beta}\right|\right]\right\}\left.\right|_{\infty} ^{x / t} \\
& =\frac{-A x}{\beta^{2}\left(V^{2}-\beta^{2}\right)} \\
& \times \operatorname{Re}\left\{\frac{-\sqrt{V^{2}-\tau^{2}}}{V^{2} \tau}-\frac{\beta}{V^{3}} \ln \left|\frac{V+\sqrt{V^{2}-\tau^{2}}}{\tau}\right|\right.
\end{aligned}
$$

$$
\begin{aligned}
& +\frac{1}{2 V \beta} \ln \left|\frac{V+\sqrt{V^{2}-\tau^{2}}}{V-\sqrt{V^{2}-\tau^{2}}}\right| \\
& -\frac{1}{2 \sqrt{V^{2}-\beta^{2}}}\left(\frac{1}{\tau}-\frac{1}{\beta}\right) \\
& \left.\times \ln \left|\frac{V^{2}-\beta \tau-\sqrt{\left(V^{2}-\beta^{2}\right)\left(V^{2}-\tau^{2}\right)}}{V^{2}-\beta \tau+\sqrt{\left(V^{2}-\beta^{2}\right)\left(V^{2}-\tau^{2}\right)}}\right| \cdot\right\}\left.\right|_{\infty} ^{x / t} \\
& =\frac{A}{\beta^{2}\left(V^{2}-\beta^{2}\right)} \\
& \times\left[\frac{\sqrt{V^{2} t^{2}-x^{2}}}{V^{2}}+\frac{\beta x}{V^{3}} \ln \left|\frac{V t+\sqrt{V^{2} t^{2}-x^{2}}}{x}\right|\right. \\
& -\frac{x}{2 V \beta} \ln \left|\frac{V t+\sqrt{V^{2} t^{2}-x^{2}}}{V t-\sqrt{V^{2} t^{2}-x^{2}}}\right| \\
& +\frac{(x-\beta t)}{2 \beta \sqrt{V^{2}-\beta^{2}}} \\
& \left.\times \ln \left|\frac{V^{2} t-\beta x+\sqrt{\left(V^{2}-\beta^{2}\right)\left(V^{2} t^{2}-x^{2}\right)}}{V^{2} t-\beta x-\sqrt{\left(V^{2}-\beta^{2}\right)\left(V^{2} t^{2}-x^{2}\right)}}\right| .\right] \\
& =\frac{A}{\beta^{2}\left(V^{2}-\beta^{2}\right)} \\
& \times\left[\frac{\sqrt{V^{2} t^{2}-x^{2}}}{V^{2}}+\frac{(x-\beta t)}{2 \beta \sqrt{V^{2}-\beta^{2}}}\right. \\
& \left.\times \ln \left|\frac{V^{2} t-\beta x+\sqrt{\left(V^{2}-\beta^{2}\right)\left(V^{2} t^{2}-x^{2}\right)}}{V^{2} t-\beta x-\sqrt{\left(V^{2}-\beta^{2}\right)\left(V^{2} t^{2}-x^{2}\right)}}\right| .\right] \\
& |x|<V t .
\end{aligned}
$$

After integrating the third nape of (31) with respect to variable $\tau, W_{3}(\tau)$ can be obtained in terms of literature [23] as

$$
\begin{aligned}
W_{3}(\tau) & =\frac{-1}{\beta} \int \frac{A}{\tau^{2}\left(V^{2}-\tau^{2}\right)^{3 / 2}} d \tau \\
& =-\frac{A}{V^{4} \beta}\left(-\frac{\sqrt{V^{2}-\tau^{2}}}{\tau}+\frac{\tau}{\sqrt{V^{2}-\tau^{2}}}\right)+C .
\end{aligned}
$$

The crack expands along the $x$-axis, so $W_{3}(\tau)$ can be performed in the definite integral, and we also take constant $C=0$. Then inserting (36) into (6), (5), the zonal displacement $v_{3}$ is acquired as

$$
\begin{aligned}
v_{3} & =\int_{0}^{t} \operatorname{Re} W_{3}(\tau) d t=\operatorname{Re} \int_{\infty}^{x / t}-\frac{x}{\tau^{2}} W_{3}(\tau) d \tau \\
& =\operatorname{Re} \int_{\infty}^{x / t}-\frac{x}{\tau^{2}} \cdot \frac{-A}{V^{4} \beta}\left(\frac{\tau}{\sqrt{V^{2}-\tau^{2}}}-\frac{\sqrt{V^{2}-\tau^{2}}}{\tau}\right) d \tau
\end{aligned}
$$




$$
\begin{array}{r}
=\left.\frac{A x}{2 V^{4} \beta} \operatorname{Re}\left(\frac{\sqrt{V^{2}-\tau^{2}}}{\tau^{2}}-\frac{3}{V} \ln \frac{V+\sqrt{V^{2}-\tau^{2}}}{\tau}\right)\right|_{\infty} ^{x / t} \\
=\frac{A t}{2 V^{4} \beta} \frac{\sqrt{V^{2} t^{2}-x^{2}}}{x}-\frac{3 A x}{2 V^{5} \beta} \ln \left|\frac{V t+\sqrt{V^{2} t^{2}-x^{2}}}{x}\right|, \\
|x|<V t .
\end{array}
$$

The displacement $v$ is the sum of divisional displacement: $v=v_{1}+v_{2}+v_{3}$. After the summation of (33), (35), and (37), the displacement $v$ is gained:

$$
\begin{aligned}
& v=\frac{A}{\beta^{2}\left(V^{2}-\beta^{2}\right)} \\
& \times\left[\frac{\sqrt{V^{2} t^{2}-x^{2}}}{V^{2}}+\frac{\beta x}{V^{3}} \ln \left|\frac{V t+\sqrt{V^{2} t^{2}-x^{2}}}{x}\right|\right. \\
& -\frac{x}{2 V \beta} \ln \left|\frac{V t+\sqrt{V^{2} t^{2}-x^{2}}}{V t-\sqrt{V^{2} t^{2}-x^{2}}}\right| \\
& +\frac{(x-\beta t)}{2 \beta \sqrt{V^{2}-\beta^{2}}} \\
& \left.\times \ln \left|\frac{V^{2} t-\beta x+\sqrt{\left(V^{2}-\beta^{2}\right)\left(V^{2} t^{2}-x^{2}\right)}}{V^{2} t-\beta x-\sqrt{\left(V^{2}-\beta^{2}\right)\left(V^{2} t^{2}-x^{2}\right)}}\right| .\right] \\
& -\frac{2 A}{V^{4} \beta^{2}} \sqrt{V^{2} t^{2}-x^{2}}+\frac{A t}{V^{3} \beta^{2}} \ln \left|\frac{V t+\sqrt{V^{2} t^{2}-x^{2}}}{x}\right| \\
& +\frac{A t}{2 V^{4} \beta} \frac{\sqrt{V^{2} t^{2}-x^{2}}}{x}-\frac{3 A x}{2 V^{5} \beta} \ln \left|\frac{V t+\sqrt{V^{2} t^{2}-x^{2}}}{x}\right| \\
& =\frac{A}{\beta}\left\{\left[\frac{1}{\left(V^{2}-\beta^{2}\right) V^{2} \beta}-\frac{2}{V^{4} \beta}+\frac{t}{2 V^{4} x}\right] \sqrt{V^{2} t^{2}-x^{2}}\right. \\
& -\frac{x}{2 V\left(V^{2}-\beta^{2}\right) \beta^{2}} \ln \left|\frac{V t+\sqrt{V^{2} t^{2}-x^{2}}}{V t-\sqrt{V^{2} t^{2}-x^{2}}}\right| \\
& +\frac{(x-\beta t)}{2 \beta^{2}\left(V^{2}-\beta^{2}\right)^{3 / 2}} \\
& \left.\times \ln \left|\frac{V^{2} t-\beta x+\sqrt{\left(V^{2}-\beta^{2}\right)\left(V^{2} t^{2}-x^{2}\right)}}{V^{2} t-\beta x-\sqrt{\left(V^{2}-\beta^{2}\right)\left(V^{2} t^{2}-x^{2}\right)}}\right| .\right] \\
& +\left[\frac{x}{V^{3}\left(V^{2}-\beta^{2}\right)}+\frac{t}{V^{3} \beta}-\frac{3 x}{2 V^{5}}\right] \\
& \left.\times \ln \left|\frac{V t+\sqrt{V^{2} t^{2}-x^{2}}}{x}\right|\right\}, \quad|x|<V t .
\end{aligned}
$$

Then substituting $|x|=\alpha t$ into (38), the bridging fibrous fracture speed $\alpha$ of fiber concrete can be readily attained, while $V, \beta$, and $t$ are also referred to as known constants, where $\Delta$ is also determined by single-axis tensile test of
TABLE 1: Relevant numerical values between dynamic stress intensity factor $K_{1}(t)$ and time $t$.

\begin{tabular}{lccccc}
\hline$t / \mathrm{ms}$ & 2 & 4 & 6 & 8 & 10 \\
$K_{1}(t) \times 10^{6} / \mathrm{N} \cdot \mathrm{m}^{-3 / 2}$ & 7.4141 & 5.2426 & 4.2806 & 3.7071 & 3.3157 \\
\hline$t / \mathrm{ms}$ & 12 & 14 & 16 & 18 & 20 \\
$K_{1}(t) \times 10^{6} / \mathrm{N}^{-3} \mathrm{~m}^{-3 / 2}$ & 3.0268 & 2.8023 & 2.6213 & 2.4714 & 2.3446 \\
\hline
\end{tabular}

TABLE 2: Relevant numerical values between dynamic stress intensity factor $K_{1}(t)$ and time $t$.

\begin{tabular}{lccccc}
\hline$t / \mathrm{ms}$ & 2 & 4 & 6 & 8 & 10 \\
$K_{1}(t) \times 10^{-4} / \mathrm{N} \cdot \mathrm{m}^{-3 / 2}$ & 0.9380 & 1.3265 & 1.6246 & 1.8759 & 2.0973 \\
\hline$t / \mathrm{ms}$ & 12 & 14 & 16 & 18 & 20 \\
$K_{1}(t) \times 10^{-4} / \mathrm{N} \cdot \mathrm{m}^{-3 / 2}$ & 2.2975 & 2.4816 & 2.6529 & 2.8139 & 2.9661 \\
\hline
\end{tabular}

bridging fibers of concrete in terms of same ways of the first problem studied.

\section{Depiction of Dynamic Stress Intensity Factor}

Analytical solutions need transforming into numerical solutions in the light of real situation of idiographic problems, hence variable law of dynamic stress intensity factor can be denoted better. The corresponding parameters [4] are put into (18) and (28) to plot $K_{1}(t)$ as a function of time $t$, respectively, and the numerical solutions of them are facilely obtained. The correlative parameters are given as follows [4]:

$$
\begin{aligned}
& E=3.3 \times 10^{10} \mathrm{~N} / \mathrm{m}^{2}\left(3.3 \times 10^{4} \mathrm{~N} / \mathrm{mm}^{2}\right), \\
& \nu=0.17, K_{\mathrm{IC}}=1.1979 \times 10^{6} \mathrm{~N} / \mathrm{m}^{3 / 2}\left(37.88 \mathrm{~N} / \mathrm{mm}^{2}\right), \\
& \rho=2400 \times 9.8 \mathrm{~N} / \mathrm{m}^{3}, V=800 \mathrm{~m} / \mathrm{s}, \quad P=600 \mathrm{~N} .
\end{aligned}
$$

Known from (18), dynamic stress intensity factor $K_{1}(t)$ decays gradually to slow down and has obvious singularity, because sole variable $t$ locates in the denominator of this expression; moreover the rest quantities are regarded as real constants, and such a trend is expressed by the curve in Figure 4. This mutative current is similar to the result of pieces of Literature. $[21,24]$. The correlative numerical value relationships are represented in Table 1.

In terms of (28), dynamic stress intensity factor $K_{1}(t)$ increases gently from zero and even reaches or exceeds fracture toughness of this material because variable $t$ lies in its numerator; this result leads to the structural instability, as depicted in Figure 5. Such a trend is homogeneous to the aftermath in pieces of literature $[25,26]$; therefore it is right. The relative numerical value relations are illuminated in Table 2.

At $V \rightarrow 0, t \rightarrow \infty$, we will have $V t \rightarrow a$; here $a$ is the length of static crack, and the above dynamic solutions can be translated into static solutions [4].

At $V \rightarrow 0, t \rightarrow \infty$, dynamic stress intensity factor of the two problems researched is less than fracture toughness $K_{1 C}$ of this kind of concrete; consequently the structure is safe. 


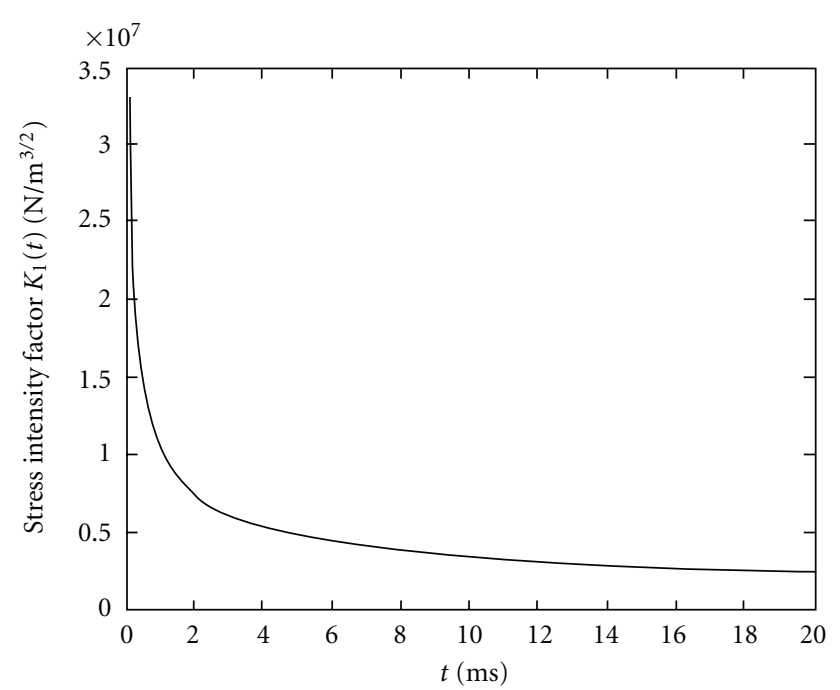

FIgURE 4: Dynamic stress intensity factors versus time.

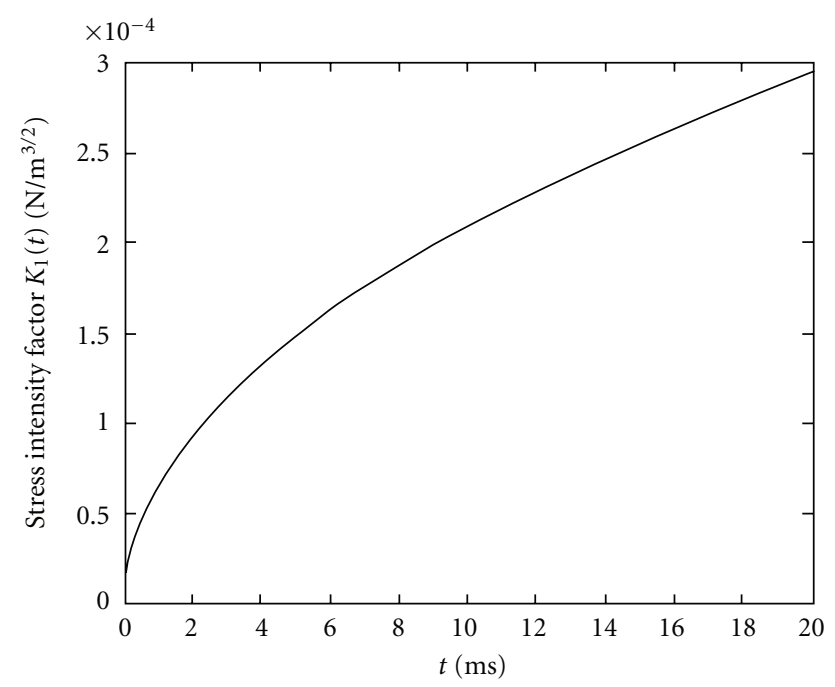

Figure 5: Dynamic stress intensity factors versus time.

\section{Conclusion}

Analytic solutions of the dynamic crack model of fiber reinforced concrete were found by the measures of complex variable theory. The approach developed in this paper based on the methods of the self-similar functions makes it conceivable to acquire the concrete solution of fiber reinforced concrete and bridging fibrous fracture speed $\alpha$. This is regarded as the analogous class of dynamic problem of the elasticity theory. However, the present solution appears to be the most straightforward and intuitive of all alternative approaches appeared up to now. Indeed, we have succeeded in a mixed Keldysh-Sedov boundary value problem on a halfplane. The problem is of adequate factual interest, since all of the members of structures in which fractures may expand are of finite dimensions and are frequently in the modality of long strips. The measure of solution is based exclusively on techniques of analytical function theory and is simple and compendious. By making some observations regarding the solution of the mixed boundary value problem we have reasonably decreased the amount of the calculative work needed to resolve such a crack propagation problem. The approaches of self-similar functions are still applicable in researches of mode I semi-infinite crack [22, 27], mode III crack [23, 28-31], and mode III interface crack [32-38] as well as axially crack $[10,39-41]$.

Utilizing the representation $f(x, y, t)=t^{n} f(x / t, y / t)$, where $n$ is an integral number, the problem can be transformed into homogeneous function of $x$ and $t$ of zeroth dimension, namely, self-similar function. This function being the analytical solution can be gained by (4), (5), (6), and (7) with homogeneous function of variable $\tau$. This method can be used in elastodynamics [10-13, 22, 27-39], in elastostatics $[14,17,42,43]$ and even in other situations $[15,43,44]$.

\section{References}

[1] T. Q. Lu and J. J. Long, "The application of synthetic fibers in concrete," Journal of Soochow University Engineering Science Edition, vol. 25, no. 2, pp. 39-42, 2005 (Chinese).

[2] G. Yong-chang and Z. Hong-Zhou, "Research and engineering applications of fiber-reinforced concrete," Guangdong Building Materials, vol. 7, pp. 8-9, 2004 (Chinese).

[3] W. Zhan-Qiao, Testing research on the fracture properties of fiber reinforced high-strength concrete, M.S. thesis, Zhengzhou University, 2004.

[4] C. Min and C. Si-Wei, "Mode I fracture of concrete or FRC," Engineering Mechanics, vol. 16, no. 4, pp. 54-58, 1999.

[5] S. Rong-Xi, W. Zhang-shui, and C. Yu-zhong, Fiber-Reinforced Cement and Riber-Reinforced Concrete, Chemical Industry Publishing House, Beijing, China, 2006.

[6] S. Mindess, J. Francis Young, and D. Darwin, Concrete, Chemical Industry Publishing House, Beijing, China, 2005.

[7] C. Ying, J. Hong-Dao, Z. Wei-Xuan, and F. Xin-Quan, "Fracture analysis of hybrid fiber reinforced cementitius composites," Joumal of Hehai University, vol. 33, no. 5, pp. 571-574, 2005.

[8] Z. Luo, X. B. Li, and T. H. Ling, "Study on the reinforcement mechanism and fracture mechanics model of steel fiber reinforced concrete," Mining Research and Development, vol. 23, no. 4, pp. 18-22, 2003 (Chinese).

[9] Z. Hong-Zhou, "Study on enhence mechanism of fiber reinforced concrete," Guangdong Water Resources and Hydropower, no. 6, pp. 13-14, 2005 (Chinese).

[10] G. P. Charepanov, Mechanics of Brittle Fracture, Nauka, Moscow, Russia, 1973.

[11] N. C. Lű, J. Cheng, and Y. H. Cheng, "Models of fracture dynamics of bridging fiber pull-out of composite materials," Mechanics Research Communications, vol. 32, no. 1, pp. 1-14, 2005.

[12] N. C. Lú, Y. H. Cheng, H. M. Xu, J. Cheng, and L. Q. Tang, "Dynamic crack models on problem of bridging fiber pulling out of composite materials," Applied Mathematics and Mechanics, vol. 25, no. 10, pp. 1194-1202, 2004.

[13] C. Jin, "Problems on elastodynamics of some orthotropic anisotropic bodies," Journal of Harbin Institute of Technology, supplement, pp. 8-21, 1985 (Chinese).

[14] N. I. Muskhelishvili, Some Fundamental Problems in the Mathematical Theory of Elasticity, Nauka, Moscow, Russia, 1966. 
[15] N. I. Muskhelishvili, Singular Integral Equations, Nauka, Moscow, Russia, 1968.

[16] R. F. Hoskins, Generalized Functions, Ellis Horwood, 1979.

[17] F. D. Gahov, Boundary-Value Problems. Fitzmatigiz, Nauka, Moscow, Russia, 1963.

[18] W. Xie-Shan, Singular Functions and Their Applications in Mechanics, Scientific Press, Beijing, China, 1993.

[19] G. C. Sih, Mechanics of Fracture4. Elastodynamics Crack Problems, Noordhoff, Leyden, Mass, USA, 1977.

[20] R. P. Kanwal and D. L. Sharma, "Singularity methods for elastostatics," Journal of Elasticity, vol. 6, no. 4, pp. 405-418, 1976.

[21] A. S. Kobayashi, "Dynam ic fracture analysis by dynam ic finite element method: generation and prediction analyses," in Nonlinear and Dynarnic Fracture Mechanics, vol. 35 of AMD, pp. 19-36, ASME, New York, NY, USA, 1979.

[22] N. C. Lü, X. G. Li, Y. H. Cheng, and J. Cheng, "Fracture dynamics problem on mode I semi-infinite crack," Archive of Applied Mechanics, vol. 81, no. 8, pp. 1181-1193, 2011.

[23] Y. H. Wang, Y. K. Cheung, and C. W. Woo, "Anti-plane shear problem for an edge crack in a finite orthotropic plate," Engineering Fracture Mechanics, vol. 42, no. 6, pp. 971-976, 1992.

[24] J. F. Kalthof, J. Beinert, and S. Winkler, "Measurements of dynamic stress intensity factors for fastrunning and arresting cracks in double-cantilever-beam specimens," in Fast Fracture and Crack Arrest, vol. STP 627 of ASTM, pp. 161-176, Philadelphia, Pa, USA, 1977.

[25] K. Ravi-Chandar and W. G. Knauss, "An experimental investigation into dynamic fracture: III. On steady-state crack propagation and crack branching," International Journal of Fracture, vol. 26, no. 2, pp. 141-154, 1984.

[26] K. Ravi-Chandar and W. G. Knauss, "An experimental investigation into dynamic fracture: IV. On the interaction of stress waves with propagating cracks," International Journal of Fracture, vol. 26, no. 3, pp. 189-200, 1984.

[27] N. C. Lü, Y. H. Cheng, X. G. Li, and J. Cheng, "Dynamic propagation problem of mode i semi-infinite crack subjected to superimpose loads," Fatigue and Fracture of Engineering Materials and Structures, vol. 33, no. 3, pp. 141-148, 2010.

[28] N. C. Lü, Y. H. Cheng, X. G. Li, and J. Cheng, "Dynamic propagation problems concerning surfaces of asymmetrical mode III crack subjected to moving loads," Applied Mathematics and Mechanics, vol. 29, no. 10, pp. 1279-1290, 2008.

[29] N. C. Lü, Y. H. Cheng, Y. T. Wang, and J. Cheng, "Dynamic fracture of orthotropic solids under anti-plane shear loading," Mechanics of Advanced Materials and Structures, vol. 17, no. 3, pp. 215-224, 2010.

[30] N. C. Lü, Y. H. Cheng, Y. T. Wang, and J. Cheng, "Dynamic extension problems concerning asymmetrical mode III crack," Applied Mathematical Modelling, vol. 35, pp. 2499-2507, 2011.

[31] N. C. Lü, Y. H. Cheng, Y. T. Wang, and J. Cheng, "Fracture dynamics problems of orthotropic solids under anti-plane shear loading," Nonlinear Dynamics, vol. 63, no. 4, pp. 793806, 2011.

[32] Y. S. Wang and D. Wang, "Transient motion of an interface dislocation and self-similar propagation of an interface crack: anti-plane motion," Engineering Fracture Mechanics, vol. 55, no. 5, pp. 717-725, 1996.

[33] K. C. Wu, "Transient motion of an interfacial line force or dislocation in an anisotropic elastic bimaterial," International Journal of Solids and Structures, vol. 40, no. 8, pp. 1811-1823, 2003.
[34] C. Atkinson, "On the dynamic stress and displacement field associated with a crack propagating across the interface between two media," International Journal of Engineering Science, vol. 13, no. 5, pp. 491-506, 1975.

[35] N. C. Lü, D. N. Yang, Y. H. Cheng, and J. Cheng, "Asymmetrical dynamic propagation problems on mode III interface crack," Applied Mathematics and Mechanics, vol. 28, no. 4, pp. 501-510, 2007.

[36] N. C. Lu, Y. H. Cheng, X. B. Tian, and J. Cheng, "Dynamic propagation problem on Dugdale model of mode III interface crack," Applied Mathematics and Mechanics, vol. 26, no. 9, pp. 1212-1221, 2005.

[37] N. C. Lü, Y. H. Cheng, X. G. Li, and J. Cheng, "Asymmetrical dynamic propagation problems concerning mode III interface crack," Composite Interfaces, vol. 17, no. 1, pp. 37-48, 2010.

[38] N. C. Lu, Y. H. Cheng, and J. Cheng, "Dynamic propagation problems concerning asymmetrical mode III interface crack," International Journal of Computational Methods in Engineering Science and Mechanics, vol. 9, no. 4, pp. 246-253, 2008.

[39] G. P. Charepanov and E. F. Afanasov, "Some dynamic problems of the theory of elasticity-A review," International Journal of Engineering Science, vol. 12, no. 5, pp. 665-690, 1970.

[40] G. P. Charepanov, Mechanics of Brittle Fracture, Nauka, Moscow, Russia, 1973.

[41] N. C. Lü, J. Cheng, and Y. H. Cheng, "Self-similar solutions of fracture dynamics problems on axial symmetry," Applied Mathematics and Mechanics, vol. 22, no. 12, pp. 1429-1435, 2001.

[42] N. I. Sneddon, Fourier Transform, McGraw-Hill, New York, NY, USA, 1951.

[43] N. I. Muskhelishvili, Some Basic Problems from the Mathematical Theory of Elasticity, P. Noordoff, Groningen, The Netherlands, 1953.

[44] L. A. Galin, Contact Problems in Elasticity Theory, GITTL, Moscow, Russia, 1953. 

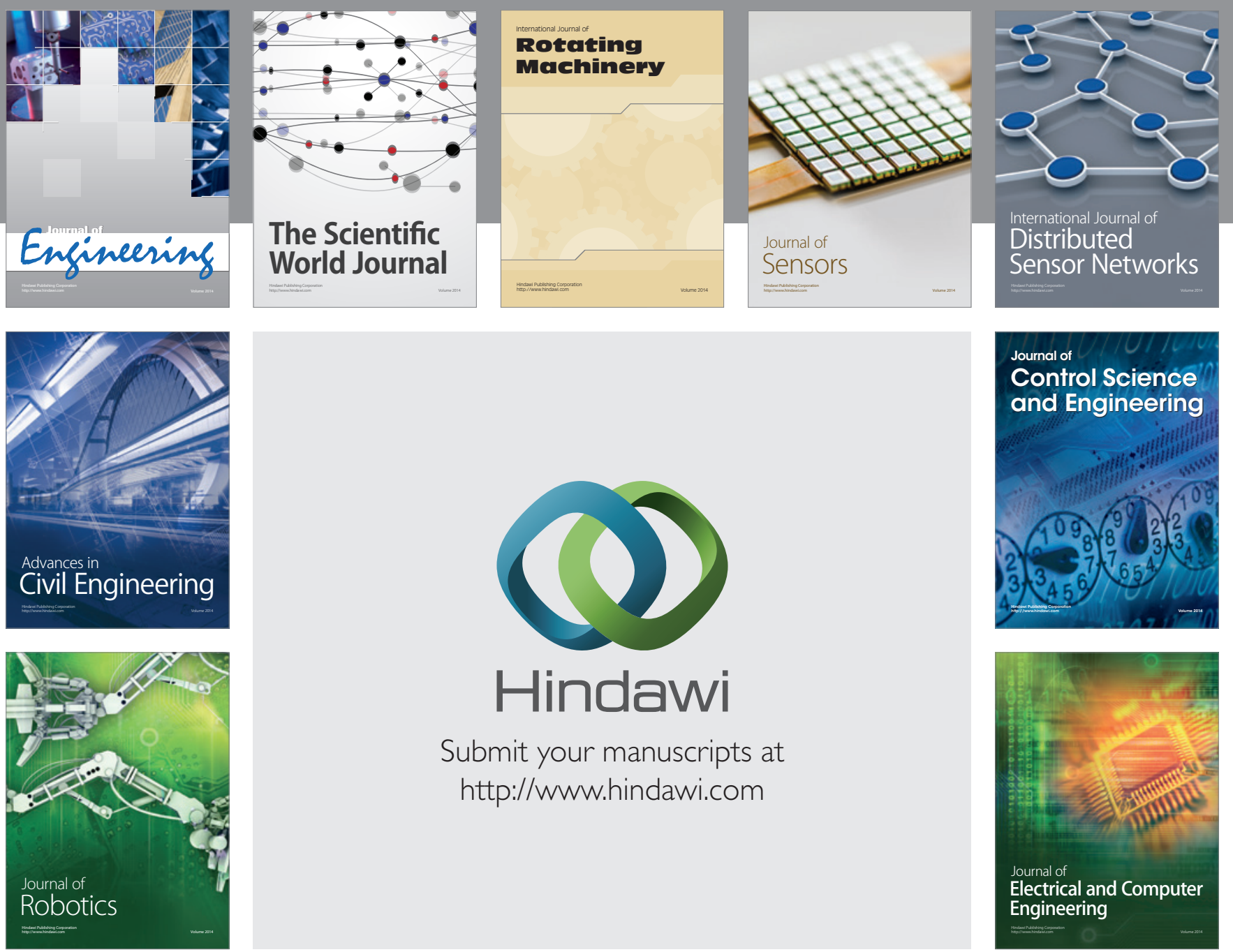

Submit your manuscripts at

http://www.hindawi.com
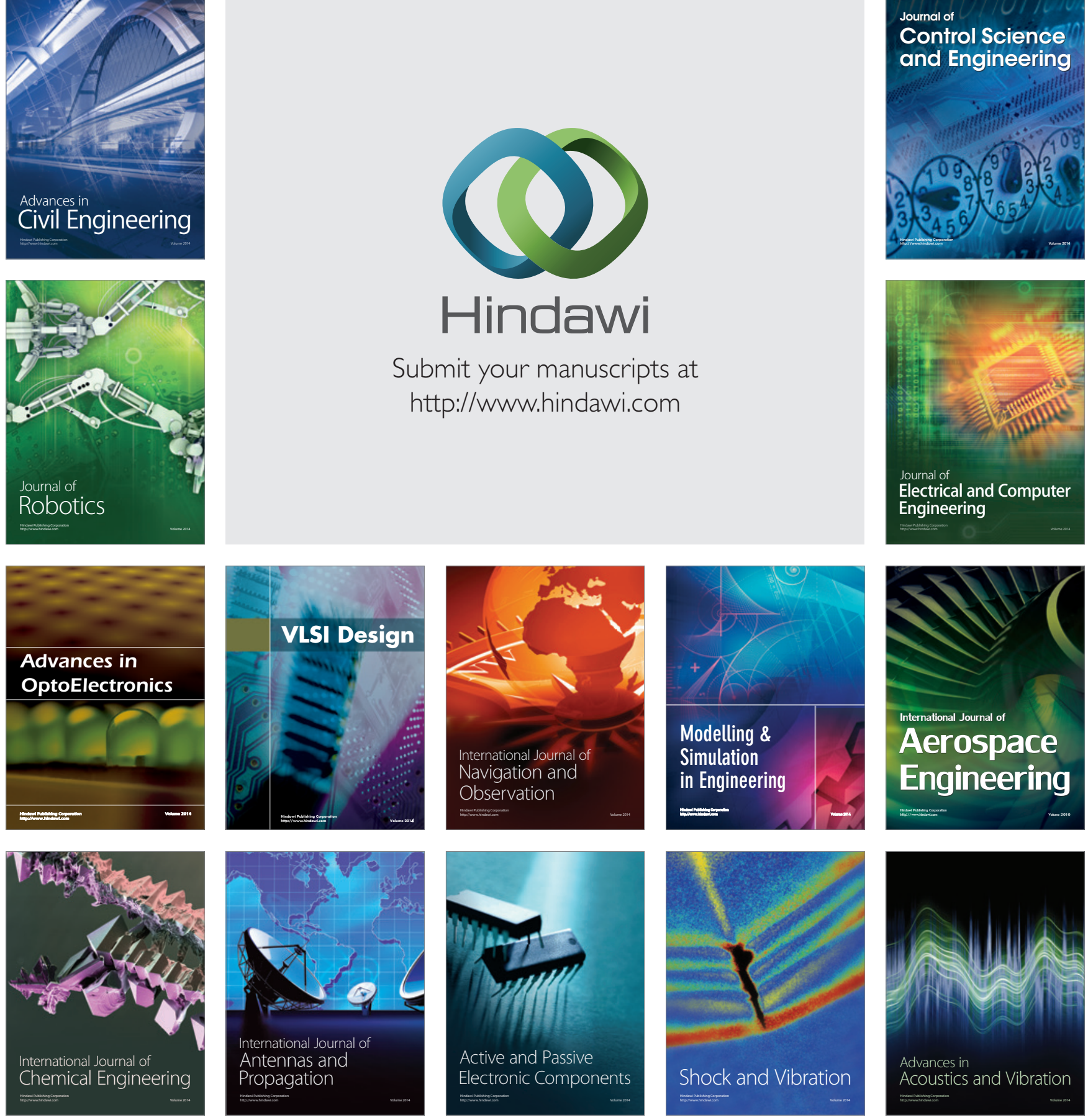